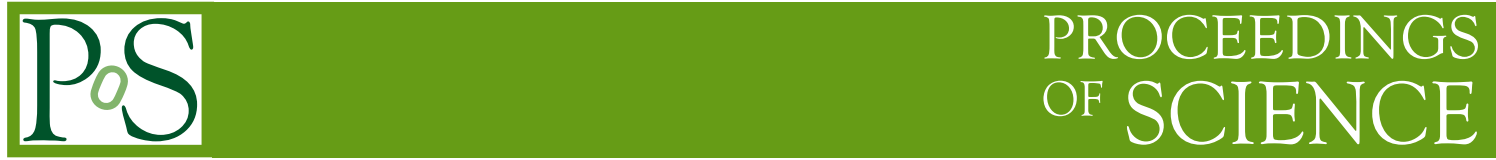

\title{
Theoretical strategies for epsilon'/epsilon
}

\section{Norman Christ* Columbia University, USA}

E-mail: nhc@phys.columbia.edu

\section{RBC and UKQCD Collaborations}

We review the current status of calculations of the two pion decays of the kaon using the firstprinciples methods of lattice gauge theory and the significant challenges that these calculations pose. While a calculation with controlled errors at even the 10-20\% level has not yet been performed, present results suggest that such a calculation of the real and imaginary parts of the $\Delta I=3 / 2$ amplitude should be accomplished within the next two years. The more difficult $\Delta I=1 / 2$ amplitude may also be now within reach.

2009 KAON International Conference KAON09,

June 09 - 122009

Tsukuba, Japan

* Speaker. 


\section{Introduction}

The two pion decays of the $\mathrm{K}$ meson have been an important topic in particle physics for more than fifty years. The same processes that lead to the discovery of $\mathrm{P}, \mathrm{C}$ and $\mathrm{CP}$ violation today hold the promise of revealing the first insights into physics beyond the standard model. For such promise to be achieved, theoretical calculations must be realized whose precision matches those of the impressive experimental results presented at this conference. The present theoretical challenge is the calculation of the hadronic matrix elements of four-quark operators which describe the relevant electro-weak processes at the relatively long distances which characterize the initial and final kaon and pion states. This current focus on the effects of low energy QCD is the result of critical work over the past thirty years using perturbative methods to analyze these processes at short distance. The result of this work is a low energy effective Hamiltonian of the form:

$$
\mathscr{H}_{\text {eff }}^{\Delta S=1}=\frac{G_{F}}{\sqrt{2}}\left\{\sum_{i=1}^{10}\left[V_{u d} V_{u s}^{*} z_{i}(\mu)-V_{t d} V_{t s}^{*} y_{i}(\mu)\right] Q_{i}\right\}
$$

where we use the notation of Ref. [1]. In Eq. 1.1 the top, bottom and charm quarks are treated as heavy and their effects have been incorporated using QCD perturbation theory so that the fourquark operators $Q_{i}$ contain only three light quarks $u, d$ and $s$.

Thus, the problem which this talk addresses is the calculation of the matrix elements of $\mathscr{H}_{\text {eff }}^{\Delta S=1}$ between an initial kaon state and a final state of two pions in either the $\mathrm{I}=0$ or I=2 state: $\left\langle\pi \pi(I)\left|\mathscr{H}_{\text {eff }}^{\Delta S=1}\right| K\right\rangle_{I=0,2}$. In principle, lattice QCD is ideally suited for such a calculation. Euclidean space lattice techniques should permit the calculation of such matrix elements without ad hoc assumptions and with numerical control of all errors. We will now discuss the difficulties posed by such calculations and the techniques which are expected to overcome them.

\section{Operator mixing and renormalization}

The 4-quark operators $Q_{i}$ which appear in Eq. 1.1 are each of dimension six. They are linear combinations of seven independent operators, which naturally divide into three groups: a single $(27,1)$ operator, two $(8,8)$ operators and four $(8,1)$ operators. The operators within each group mix under renormalization as well as with operators of lower dimension. Such a renormalization pattern is well understood for continuum operators and can be managed using standard techniques if a chirally invariant regulator, such as dimensional regularization is employed.

For a lattice calculation, these operators must now be defined using a lattice regulator. Given the essential role played by chiral symmetry in limiting the number of operators which can appear and their mixing, it is essential to use a lattice fermion formulation which respects chiral symmetry. The domain wall fermion (DWF) formulation has the needed chiral symmetry with violations that can be made arbitrarily small as size in the fifth dimension $\left(L_{s}\right)$ is increased. This formulation was used in the first complete quenched calculations of the $K \rightarrow|0\rangle$ and $K \rightarrow \pi$ matrix elements of these ten operators $Q_{i}$. [1, 2]. Now the RBC/UKQCD and LHPC collaborations have created substantial ensembles for $2+1$ flavor QCD for a variety of light quark masses and two lattice spacings. These configurations combined with new larger volume, larger lattice spacing configurations will provide an excellent foundation for a correctly unitary, full QCD calculation of these decay amplitudes. 
Even with such a chiral lattice formulation, we must still relate the lattice operators with the equivalent continuum operators appearing in Eq. 1.1. Such a matching between continuum and lattice operators can be accurately carried out using the regularization-independent, Rome/Southampton RI/MOM scheme, which can be applied to both continuum and lattice operators.

The strength of the RI/MOM scheme is that it can be applied to lattice operators non-perturbatively by imposing simple conditions on off-shell gauge-fixed Greens functions evaluated using standard lattice gauge theory techniques. In fact, these techniques have been successfully used to transform matrix elements of the bare lattice operators $Q_{i}^{\text {lat }}$ into those of RI/MOM-normalized operators in both quenched [3, 1] and full QCD [4] calculations. This method is increasingly well understood [5], with improved techniques giving statistical errors on the percent level. At present the largest errors, on the 5\% level, come from the use of perturbation theory to connect the RI/MOM and $\overline{\mathrm{MS}}$ schemes. We conclude that the problems of operator normalization and mixing are under adequate control and pose no special difficulties for the topic at hand.

\section{Quadratic divergence}

A cause for concern when considering a calculation of the $\Delta I=1 / 2 K \rightarrow \pi \pi$ amplitude is the presence of quadratically divergent terms. In a lattice calculation such terms are finite but larger than the physical amplitude by a factor proportional to $1 / a^{2}$. While such terms will not contribute to any properly constructed physical amplitude, their removal, either by explicit subtraction or by the averaging of the relevant matrix element to zero may increase statistical or to amplify systematic errors.

For example, in the standard calculation of the matrix element of the operator $Q_{6}$ using leading order chiral perturbation theory (LO ChPT) a combination of $K \rightarrow \pi$ and $K \rightarrow|0\rangle$ amplitudes is required in which the later can be viewed as subtracting the quadratic divergence from the former. These two amplitudes and their much smaller difference is shown in Fig. 1. As can be seen this subtraction reduces the amplitude by nearly a factor of ten but still gives a result with few percent statistical errors. These large matrix elements also enhance the systematic errors associated with finite $L_{s}$. However, it can be shown $[6,7]$ that such errors are at most on the few percent level.

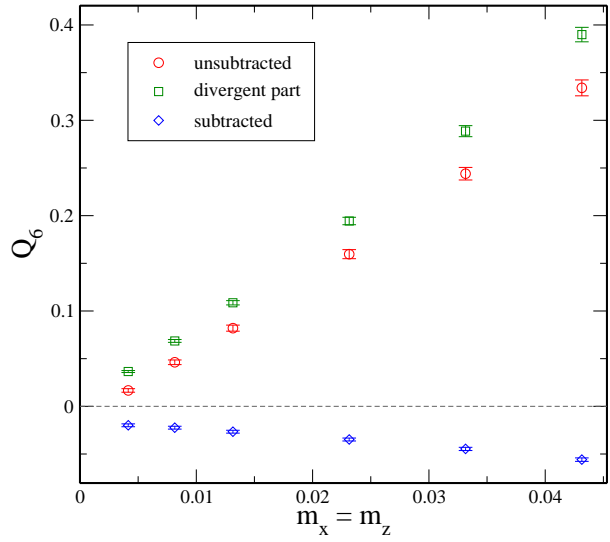

Figure 1: Matrix elements of $Q_{6}$ (circles) which contain a quadratic divergence, the subtraction term (squares) and their difference (diamonds).

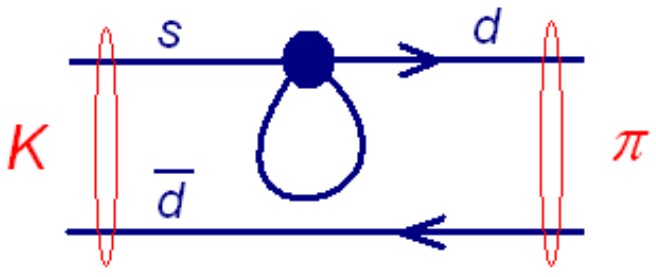

Figure 2: Example graph containing a quadratically divergent quark loop which appears in the $\left\langle\pi\left|Q_{6}\right| K\right\rangle$ matrix element. 
These quadratic divergences resulting from quark loops of the sort shown in Fig. 2 appear multiplying operators $\bar{s}\left(1 \pm \gamma^{5}\right) d$ which vanish when evaluated in momentum conserving $K \rightarrow \pi \pi$ matrix elements. However, such terms vanish only after an average over gauge configurations and therefore may introduce large statistical fluctuations. If necessary, an explicit subtraction can be introduced which will not affect the average but should reduce the variance to the acceptable level found in the $K \rightarrow \pi$ matrix elements in Fig. 1. Thus, experience with $K \rightarrow \pi$ calculations $[1,2]$ suggests that these $O\left(1 / a^{2}\right)$ components of the $\Delta I=1 / 2$ operators will not pose serious difficulties.

\section{Two pion final state}

An important problem associated with such $K \rightarrow \pi \pi$ calculations is summarized by the MaianiTesta theorem which points out that the large Euclidean time limit used in lattice QCD to project onto physical states will yield a $\pi-\pi$ state with zero relative momentum, not the physical state which should appear in the matrix element of interest. There are now two methods to circumvent this difficulty. The first uses $S U(3) \times S U$ (3) ChPT to relate the $K \rightarrow \pi \pi$ amplitudes of interest to simpler $K \rightarrow \pi$ and $K \rightarrow|0\rangle$ matrix elements. This avoids dealing directly with a state containing two pions but, as is discussed in Section 4.1 below, relies on ChPT in a region where its validity is highly uncertain. The second more promising method is based on finite volume techniques [8]. This second approach is discussed below in Section 4.2.

\subsection{Chiral perturbation theory}

The first quenched calculations of the complete $K \rightarrow \pi \pi$ amplitude were carried out using LO ChPT [1, 2]. While the results for the real parts of $A_{0}$ and $A_{2}$ may have been encouraging, the value of $\varepsilon^{\prime} / \varepsilon$ was near zero and slightly negative - far from the experimental value. However, Golterman and Pallante [9] discovered that the ChPT structure of the quenched and full theory was very different, with the quenched theory possessing more singular chiral logarithms than are present in the complete, unquenched theory.

Such problems with the quenched approximation can be avoided by moving to full QCD simulations and the RBC/UKQCD collaboration has repeated the earlier quenched work using $2+1$ flavor, DWF gauge ensembles [4]. In addition to the inclusion of fermion loops, this new calculation explores lighter quark masses, including "partially quenched" amplitudes with unequal valence and sea quark masses, allowing a detailed comparison with ChPT. Unfortunately, the RBC/UKQCD results for the quark mass behavior of the standard meson masses and decay constants $[10,11]$ and these detailed studies of the $K \rightarrow \pi$ and $K \rightarrow|0\rangle$ amplitudes [4] strongly suggest that ChPT does not provide a reliable description for masses as large as that of the physical $\mathrm{K}$ meson.

In Fig. 3 we show an attempt to fit next leading order (NLO) ChPT to the ratio

$$
\left\langle\pi\left|O^{(27,1)}\right| K\right\rangle / f_{K} f_{\pi} m_{\pi} m_{K}
$$

in order to extract the LEC $\alpha_{27}$. This figure reveals two difficulties. First, we are fitting the ratio shown in Eq. 4.1 because we were not able to obtain a sensible fit to the simpler matrix element in the numerator. One might argue that the NLO ChPT terms in this ratio will be reduced because

of significant cancelation between the NLO chiral logarithms which appear in the numerator and 
denominator. However, a circumstance in which we must construct artificial ratios in order to obtain sensible fits naturally raises serious doubts about the applicability of ChPT.

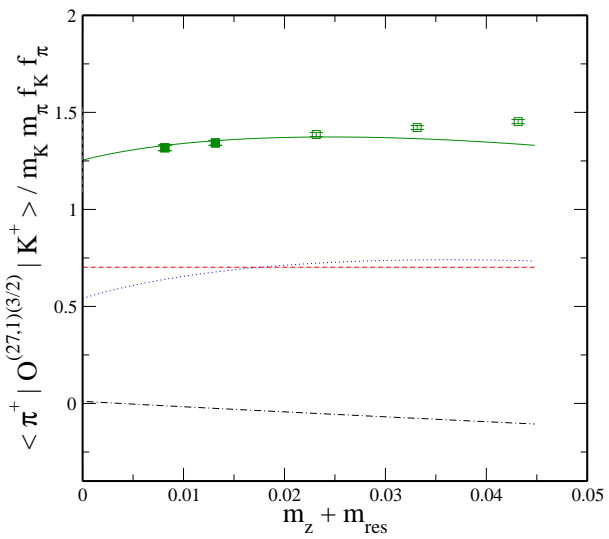

Figure 3: A NLO ChPT fit to the ratio in Eq. 4.1 compared to the lightest sea quark results. The individual components of the fit as well as their total are shown. The NLO analytic and logarithmic terms appear in the middle while the smallest contribution, near zero, is the LO term.

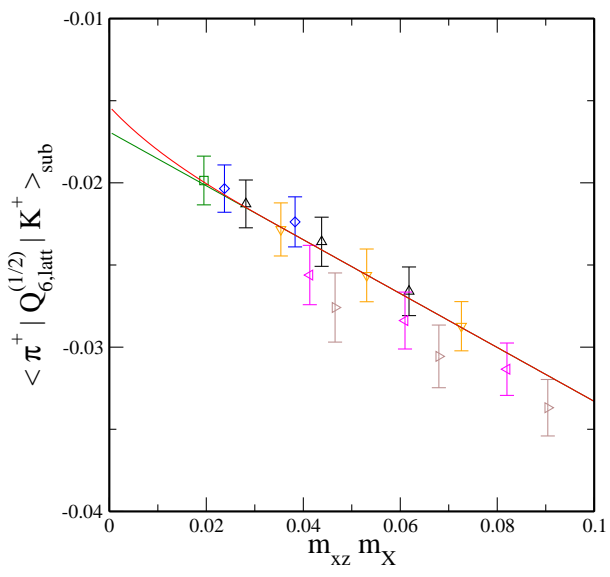

Figure 4: Results for the $(8,1)$ operator $Q_{6}$ after subtraction of the quadratic divergence together with a LO chiral fit. The small deviation shown at small quark masses is an estimate of the possible effect of a NLO chiral logarithm.

The second problem is the dominance of the next leading order (NLO) term over that of leading order. While the leading order term may be accidentally suppressed, the failure of the fit to show a standard hierarchy among the orders of the expansion suggests the more probable situation that ChPT is inapplicable and the result is a fit to an essentially arbitrary function with no ordering between the various terms. A similar situation is found for the other two $\Delta I=3 / 2,(8,8)$ operators.

The situation is less clear for the $(8,1) \Delta I=1 / 2$ operators because the NLO ChPT contains more low energy constants (LEC's) making our data inadequate to carry out a complete NLO ChPT fit. As can be seen in Fig. 4 a simple linear fit (leading order in ChPT) describes the data very well. However, as shown by the additional up-turning curve added to the left of the data points, possible NLO logarithmic behavior that is entirely consistent with our calculated points can change the slope at vanishing light quark mass by a factor of two. Since it is this slope which is the LEC of interest, $\alpha_{6}$, we must assign a $100 \%$ systematic error to our result.

In summary, while the use of the $2+1$ flavor DWF configurations has removed the uncertainties associated with the quenched approximation, the use of smaller quark masses and partial quenching has raised new concerns about the validity of ChPT in the kinematic region needed to determine the $K \rightarrow \pi \pi$ amplitudes. First, as discussed above, our calculation suggests that the needed LEC's cannot be reliably determined from our present range of masses. This problem can be addressed by moving to lighter masses. However, there is a second, more serious difficulty. Even if these LECs were known, we would still require a second application of ChPT to compute the $K \rightarrow \pi \pi$ amplitudes. This use of ChPT would involve exactly the region of large quark masses and pion momenta where the studies above suggest the theory fails. There is no solution to this problem if we chose to work in the physical world with $498 \mathrm{MeV}$ kaons. 


\subsection{Calculation with two pion final states}

We conclude that it is necessary to directly compute matrix elements using states containing two pions. Evaluating matrix elements of such a 2-particle state using Euclidean space lattice techniques is now well understood [8] but still presents serious practical challenges. The key to computing such $\pi-\pi$ matrix elements is to understand and exploit the finite volumes that necessarily appear in a lattice calculation.

While the underlying weak operator connects the initial $\mathrm{K}$ meson to two pions in an s-wave, the rectangular box of a lattice calculation mixes that $l=0$ state with states with $l=4,8, \ldots$ In the reasonable approximation that the $\pi-\pi$ phase shifts, $\delta_{l}$, vanish for $l>0$, then the weight of the $l=0$ component of the finite volume eigenstates can be computed knowing only $\delta_{l=0}$ and allowing the physical $l=0$ matrix element to be extracted from that of the finite volume state. While the finite volume $\pi-\pi$ ground state is close to threshold with nearly zero relative momentum, there are a series of excited states with relative pion momenta which differ from the free-particle multiples of $2 \pi / L$ by calculable amounts, again determined by $\delta_{0}$.

For a cubic box with $L=6 \mathrm{fm}$, the pions in the first excited state have relative momentum very close to the physical value of $p=205 \mathrm{MeV}$. With present resources, this is an inaccessibly large volume. However, this problem can be circumvented by three strategies. The first starts with a $\mathrm{K}$ meson with non-zero momentum. Momentum conservation requires that the two pion state carry this same momentum which typically implies that one pion must remain at rest. If the initial kaon has a momentum of $740 \mathrm{MeV}$, then the relative momentum of the two final pions is physical. This $740 \mathrm{MeV}$ value can be easily achieved on a practical $3 \mathrm{fm}$ lattice if $p_{K}=(1,1,1) 2 \pi / L$ or $p=\sqrt{3} 2 \pi / L$. Of course, with all methods, the challenge of an $L=6 \mathrm{fm}$ volume must eventually be met as the mass of the pions used approaches its physical value. The rule-of-thumb that $L m_{\pi} \geq 4$ to avoid finite volume effects also requires $L \geq 5.7 \mathrm{fm}$ when $m_{\pi}=138 \mathrm{MeV}$.

This $p_{K}>0$ approach has been studied by T. Yamazaki [12] for the $\Delta I=3 / 2$ amplitudes with encouraging results. However, the large momentum of the $K$ and $\pi$ is a cause for concern. Since momentum conservation takes effect only after the average over gauge fields has been performed, the amplitude on each configuration will be dominated by the much larger contributions from $K$ and $\pi$ states at rest. The large cancelation needed to give the correct averages implies a corresponding large statistical noise. Never-the-less, this method works for both the $\Delta I=3 / 2$ and $\Delta I=1 / 2$ cases and for $\Delta I=1 / 2$ automatically removes the vacuum contribution which necessarily carries zero momentum.

A second approach imposes anti-periodic boundary conditions on the pions. This is easily done for the $\Delta I=3 / 2$ amplitude with its $I=2$ final state [13] by using anti-periodic boundary conditions for the $u$ quark but periodic boundary conditions for the $d$. Isospin symmetry implies that the $A_{2}$ amplitude can be determined from a matrix element with a $\left|\pi^{+} \pi^{+}\right\rangle$final state, where both $\pi^{+}$ mesons obey anti-periodic boundary conditions and therefore carry the physical momentum $\pi / L=$ $205 \mathrm{MeV}$ when $L=3 \mathrm{fm}$, a practical requirement. For $I=2$, the final state must be constructed from valence quarks and one can argue [14] that if the anti-periodic boundary conditions are applied only to the valence quarks in the calculation, allowing standard periodic boundary condition gauge configurations, then only errors exponentially suppressed by the lattice size are introduced.

Imposing anti-periodic boundary conditions on the pions is more difficult for the $I=0$ state 
where the quark content is less controlled. An attractive method is to impose G parity boundary conditions [15] on the quarks which guarantees anti-periodic pions:

$$
\left(\begin{array}{c}
u(x) \\
d(x)
\end{array}\right) \rightarrow\left(\begin{array}{c}
\mathscr{C} \bar{d}\left(x+L \hat{e}_{i}\right) \\
-\mathscr{C} \bar{u}\left(x+L \hat{e}_{i}\right)
\end{array}\right) \rightarrow\left(\begin{array}{c}
-u\left(x+2 L \hat{e}_{i}\right) \\
-d\left(x+2 L \hat{e}_{i}\right)
\end{array}\right)
$$

where $\mathscr{C}$ is the standard $4 \times 4$ charge-conjugation matrix and $\hat{e}_{i}$ a unit vector in a direction in which these $\mathrm{G}$ parity boundary conditions are imposed.

These boundary conditions require special gauge ensembles where the gauge fields obey charge conjugate boundary conditions and the light sea quarks also obey $\mathrm{G}$ parity boundary conditions. In addition, special treatment is required for the strange quark which is best made part of a fictitious iso-doublet. These sea quarks can be represented by standard, positive-definite DWF determinants (no Pfaffians needed) although a square root of the strange quark determinant is required to keep the correct number of flavors. Given the computational costs required by the disconnected diagrams, the extra difficulty of generating these special gauge configurations may be relatively minor, making $\mathrm{G}$ parity boundary conditions an attractive approach to the $\Delta I=1 / 2$ amplitude.

\section{Disconnected diagrams}

Because the $I=0, \pi-\pi$ state has the flavor quantum numbers of the vacuum, $I=0, \pi-\pi$ propagators and $K \rightarrow \pi \pi(I=0)$ amplitudes all contain diagrams in which no quark lines connect the initial and final states. These quantities then contain a vacuum contribution which does not vanish as the separation between the source and the sink or weak decay operator grows. Such unphysical vacuum terms either vanish because of inconsistent non-flavor quantum numbers (e.g. $\vec{p} \neq 0$ ) or must be explicitly subtracted. Unfortunately, even when removed, the separation-independent fluctuations of such vacuum terms can quickly overwhelm the exponentially decreasing physical signal, presenting major problems for the lattice calculation of many interesting physical quantities.

These difficulties may be least severe for the $I=0, \pi-\pi$ system. As the quark masses become more physical and the pions less massive, the $\pi-\pi$ signal falls less rapidly with increasing time allowing a signal to be obtained at larger times. In Fig 5 we show preliminary results of $I=0, \pi-\pi$ scattering study being carried out by Qi Liu. For small time separations the disconnected amplitude can be determined and appears much smaller than the connected piece. However for times of 5 or greater, the noise in the disconnected part begins to contribute substantially to the error in the complete amplitude, severely limiting the temporal region where a plateau in the effective mass and the needed evidence for asymptotic behavior can be established.

While this preliminary work is only beginning to uncover the difficulties of dealing with the disconnected contributions to the $K \rightarrow \pi \pi(I=0)$ amplitudes, we believe there is reason for optimism. These results were obtained in a few months on 1024-node QCDOC partitions. One hundred and thirty $16^{3} \times 32$ lattice configurations were analyzed computing 32 sets of propagators on each, using wall sources located on each possible time hyperplane. With larger computer resources, working with larger lattice volumes and collecting larger statistics will be practical. While this will not permit the analysis to be extended to significantly larger times, it will yield more accurate results in the time range $0 \leq t \leq 6$ where we hope that the introduction of more interpolating opera- 

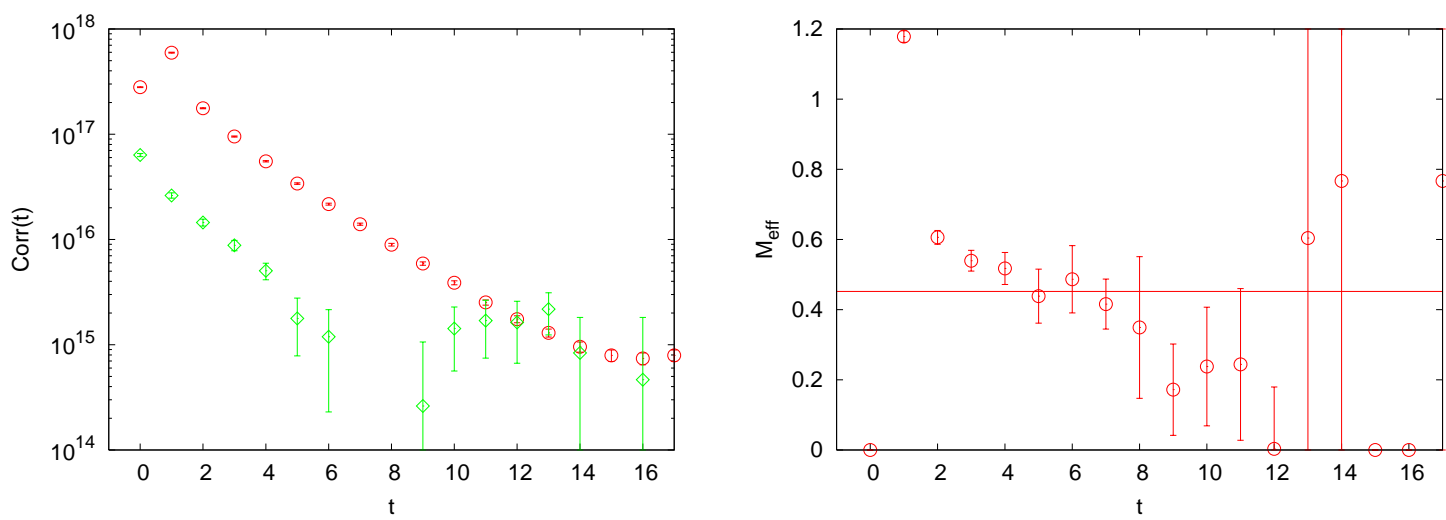

Figure 5: The left panel shows the connected (circles) and disconnected (diamonds) contributions to the $I=0 \pi-\pi$ correlator. The right-hand panel shows the resulting 2 pion effective mass. The missing points in the left panel result from negative values for the amplitude which cannot be shown on a logarithmic plot.

tors will allow the extraction of both the ground and excited states so that physical matrix elements can be determined without relying on a simple large-time limit to project onto the ground state.

\section{Outlook}

Substantial efforts over the past ten years strongly suggest that a lattice calculation of the complex $K \rightarrow \pi \pi$ amplitudes $A_{0}$ and $A_{2}$, accurate to $10-20 \%$, requires a full (unquenched) lattice QCD calculation in which on-shell two pion states are studied. This experience suggests that issues of divergent penguin graphs and operator renormalization and mixing can be treated with a few percent precision using chiral fermions and non-perturbative RI/MOM techniques. Existing finite volume methods should permit the direct evaluation of matrix elements with on-shell $|\pi \pi\rangle$ states. The disconnected diagrams which appear in the $I=0$ amplitude pose the most serious challenge which may be overcome with substantial statistics and a careful multi-state analysis of correlation functions evaluated at relatively small time separations.

Based on this assessment, computing the complex $K \rightarrow \pi \pi$ amplitudes has become an important research goal of the RBC and UKQCD collaborations. The first objective is the calculation of the $\Delta I=3 / 2$ amplitude $A_{2}$ which can be done on standard, 2+1 flavor gauge configurations using anti-periodic valence quarks. We are presently carrying out a quenched calculation on a $3.6 \mathrm{fm}$, $24^{3} \times 64$ volume [16]. We expect this to provide interesting results for matrix elements of the three $\Delta I=3 / 2$ operators and guidance for a follow-on calculation using the $2+1$ flavor, 4.6 fm lattice ensembles now being generated by the RBC/UKQCD and LHPC collaborations on the ALCF at Argonne. We expect this effort to determine $A_{2}$ to an accuracy of $10-20 \%$ within two years.

For the $\Delta I=1 / 2$ amplitudes a meaningful quenched calculation is not possible and our initial calculations are being performed on $2+1$ flavor $16^{3} \times 32$ lattice volumes with $430 \mathrm{MeV}$ pions. A first, complete $K \rightarrow \pi \pi$ calculation is now underway including all connected and disconnected diagrams. With the experience gained in this calculation, we hope to exploit the next generation of 100-teraflops sustained computers as they become available in roughly one year's time to move to larger volumes and lighter pion masses. This may allow calculations of both the $\Delta I=3 / 2$ and $1 / 2$ 
amplitudes giving $10-20 \%$ results for both the $\Delta I=1 / 2$ rule and $\varepsilon^{\prime} / \varepsilon$ in 2-3 years. While this may easily be optimistic, it is surely an exciting and increasingly realistic goal to pursue.

\section{Acknowledgments}

The author acknowledges the many ideas and insights of his RBC/UKQCD collaborators which have been summarized here and the work of Shu Li, Matthew Lightman and Qi Liu whose results were described above. This research was supported by DOE grant DE-FG02-92ER40699.

\section{References}

[1] RBC Collaboration, T. Blum et al., Kaon matrix elements and cp-violation from quenched lattice qcd. $i$ : The 3-flavor case, Phys. Rev. D68 (2003) 114506, [hep-lat / 0110075 ].

[2] CP-PACS Collaboration, J. I. Noaki et al., Calculation of non-leptonic kaon decay amplitudes from $k$ - > pi matrix elements in quenched domain-wall qcd, Phys. Rev. D68 (2003) 014501, [hep-lat/0108013].

[3] C. Dawson, Non-perturbative renormalization using domain wall fermions, Nucl. Phys. Proc. Suppl. 83-84 (2000) 854, [hep-lat/9909107].

[4] S. Li and N. H. Christ, Chiral perturbation theory, K to pi pi decays and $2+1$ flavor domain wall $Q C D, 0812.1368$.

[5] Y. Aoki et al., Non-perturbative renormalization of quark bilinear operators and $B_{K}$ using domain wall fermions, Phys. Rev. D78 (2008) 054510, [0712 . 1061].

[6] N. Christ, Domain wall fermions, approximate chiral symmetry and weak matrix elements, Domain Wall Fermions at Ten Years, Riken-BNL Research Center, March 15-17, 2007. (2007).

[7] S. R. Sharpe, Future of Chiral Extrapolations with Domain Wall Fermions, arXiv : 0706.0218 [hep-lat].

[8] L. Lellouch and M. Luscher, Weak transition matrix elements from finite-volume correlation functions, Commun. Math. Phys. 219 (2001) 31-44, [hep-lat/ 0003023 ].

[9] M. Golterman and E. Pallante, Effects of quenching and partial quenching on penguin matrix elements, JHEP 10 (2001) 037, [hep-lat / 0108010 ].

[10] RBC-UKQCD Collaboration, C. Allton et al., Physical Results from 2+1 Flavor Domain Wall QCD and SU(2) Chiral Perturbation Theory, Phys. Rev. D78 (2008) 114509, [0 804 . 0473 ].

[11] RBC/UKQCD Collaboration, P. A. Boyle, Kaon physics from lattice, Talk at this conference (2009).

[12] RBC Collaboration, T. Yamazaki, On-shell $\Delta I=3 / 2$ kaon weak matrix elements with non-zero total momentum, Phys. Rev. D79 (2009) 094506, [0 807 . 3130].

[13] C. H. Kim, Delta(i) $=3 / 2 k \rightarrow$ pi pi with physical final state, Nucl. Phys. Proc. Suppl. 140 (2005) 381-383.

[14] C. T. Sachrajda and G. Villadoro, Twisted boundary conditions in lattice simulations, Phys. Lett. B609 (2005) 73-85, [hep-lat/0411033].

[15] C.-h. Kim and N. H. Christ, $K->$ pi pi decay amplitudes from the lattice, Nucl. Phys. Proc. Suppl. 119 (2003) 365-367, [hep-lat/0210003].

[16] RBC/UKQCD Collaboration, M. Lightman, Physical matrix elements for Delta I = 3/2 channel K to pi pi decays, PoS LATTICE2008 (2008) 273, [0906 . 184 7]. 